\title{
On non-proper intersections and local intersection numbers
}

\author{
Mats Andersson ${ }^{1} \cdot$ Håkan Samuelsson Kalm ${ }^{1}$ - Elizabeth Wulcan ${ }^{1}$
}

Received: 24 March 2020 / Accepted: 24 June 2021 / Published online: 28 July 2021

(c) The Author(s) 2021

\begin{abstract}
Given equidimensional (generalized) cycles $\mu_{1}$ and $\mu_{2}$ on a complex manifold $Y$ we introduce a product $\mu_{1} \diamond_{Y} \mu_{2}$ that is a generalized cycle whose multiplicities at each point are the local intersection numbers at the point. If $Y$ is projective, then given a very ample line bundle $L \rightarrow Y$ we define a product $\mu_{1} \bullet L \mu_{2}$ whose multiplicities at each point also coincide with the local intersection numbers. In addition, provided that $\mu_{1}$ and $\mu_{2}$ are effective, this product satisfies a Bézout inequality. If $i: Y \rightarrow \mathbb{P}^{N}$ is an embedding such that $i^{*} \mathcal{O}(1)=L$, then $\mu_{1} \bullet L \mu_{2}$ can be expressed as a mean value of Stückrad-Vogel cycles on $\mathbb{P}^{N}$. There are quite explicit relations between $\diamond_{Y}$ and $\bullet_{L}$.
\end{abstract}

\section{Introduction}

Let $Y$ be a complex manifold of dimension $n$. A cycle on $Y$ is a locally finite linear combination, over $\mathbb{Z}$, of subvarieties of $Y$. Assume that $\mu_{1}$ and $\mu_{2}$ are equidimensional cycles on $Y$. If they intersect properly, i.e., the expected dimension $\rho=\operatorname{dim} \mu_{1}+\operatorname{dim} \mu_{2}-n$ is equal to the dimension of their set-theoretical intersection $V=\left|\mu_{1}\right| \cap\left|\mu_{2}\right|$, then there is a well-defined intersection cycle

$$
\mu_{1} \cdot Y \mu_{2}=\sum m_{j} V_{j}
$$

where $V_{j}$ are the irreducible components of $V$ and $m_{j}$ are integers. If $\mu_{1}$ and $\mu_{2}$ do not intersect properly, i.e., $\operatorname{dim} V>\rho$, following [7], the product $\mu_{1} \cdot Y \mu_{2}$ is represented by a cycle of dimension $\rho$ on $V$ that is determined up to rational equivalence, i.e., a Chow class on $V$. In case $Y=\mathbb{P}^{n}$ there is a construction of a product $\mu_{1} \cdot S V \mu_{2}$ due to Stückrad and Vogel, $[11,13]$, that is represented by a cycle on $V$ with components of various degrees. This cycle, which we call a SV-cycle, is obtained by a quite explicit procedure, which however

Mats Andersson

matsa@chalmers.se

Håkan Samuelsson Kalm

hasam@chalmers.se

Elizabeth Wulcan

wulcan@chalmers.se

1 Department of Mathematics, Chalmers University of Technology and the University of Gothenburg, S-412 96 Göteborg, Sweden 
involves various choices. By van Gastel's formula, [9], see also [6], one can obtain the Chow class $\mu_{1} \cdot Y \mu_{2}$ from a generic representative of $\mu_{1} \cdot S V \mu_{2}$.

In the 1990s Tworzewski, [12], introduced local intersection numbers $\epsilon_{\ell}\left(\mu_{1}, \mu_{2}, x\right)$ at each point $x, 0 \leq \ell \leq \operatorname{dim} V$, which reflect the complexity of the intersection at dimension $\ell$. In particular, if the intersection is proper, then $\epsilon_{\ell}\left(\mu_{1}, \mu_{2}, x\right)$ is the multiplicity of $\mu_{1} \cdot Y \mu_{2}$ at $x$ when $\ell=\operatorname{dim} V$ and 0 otherwise. In this case thus all these numbers are represented by the global cycle $\mu_{1} \cdot Y \mu_{2}$. In general however there is no single cycle whose multiplicities of its components of various dimensions are precisely the local intersection numbers for all points of $Y$, that is, which represents all these local intersection numbers.

Since both the local and global intersections are defined within algebraic geometry, it is natural from such a point of view to look for a way to unify these theories. However since this cannot be done by cycles it is natural to look for slightly more general geometric objects that may have the desired local multiplicities and at the same time in a reasonable sense represent the global intersection products.

To this end, in [4], together with Eriksson and Yger, we introduced, for any reduced analytic space $X$, the group $\mathcal{B}_{k}(X)$ of generalized cycles of dimension $k$, modulo a certain equivalence relation, that contains the group $\mathcal{Z}_{k}(X)$ of cycles of dimension $k$ as a subgroup. The generalized cycles classes in $\mathcal{B}_{k}(X)$ share many properties with (usual) cycles. For instance, each $\mu \in \mathcal{B}_{k}(X)$ has a well-defined (integer) multiplicity mult $x$ a at each point $x \in X$ and a Zariski support $|\mu|$. Each generalized cycle class is a unique sum of irreducible generalized cycle classes. Moreover, the generalized cycle classes $\mu$ in $\mathcal{B}_{k}(X)$ that have Zariski support $|\mu|$ on a subvariety $Z \subset X$ are naturally identified with $\mathcal{B}_{k}(Z)$; see Sect. 2.1 below for precise definitions and statements. One can think of generalized cycle classes as mean values of cycles. We let $\mathcal{B}(X)=\oplus_{0}^{m} \mathcal{B}_{k}(X)$ if $m=\operatorname{dim} X$.

For $\mu_{1}, \mu_{2} \in \mathcal{B}\left(\mathbb{P}^{n}\right)$ we defined with Eriksson and Yger, [5], an element $\mu_{1} \bullet \mu_{2} \in \mathcal{B}\left(\mathbb{P}^{n}\right)$ that is equal to $\mu_{1} \cdot \mathbb{P}^{n} \mu_{2}$ if the intersection is proper, and whose multiplicities at each point coincide with the local intersection numbers. If $\mu_{j}$ have pure dimensions and the expected dimension

$$
\rho:=\operatorname{dim} \mu_{1}+\operatorname{dim} \mu_{2}-n
$$

is non-negative, then we have the Bézout equality

$$
\operatorname{deg}\left(\mu_{1} \bullet \mu_{2}\right)=\operatorname{deg} \mu_{1} \cdot \operatorname{deg} \mu_{2} .
$$

Roughly speaking, $\mu_{1} \bullet \mu_{2}$ is defined as a mean value of SV-cycles $\mu_{1} \cdot S V \mu_{2}$ in case $\mu_{j}$ are cycles.

In this paper we introduce two global intersection products that both respect all the local intersection numbers. The first one is defined on an arbitrary complex manifold. The second one generalizes the •-product and satisfies a Bézout inequality, but it is only defined on projective manifolds.

Here is our first main theorem.

Theorem 1.1 Let $Y$ be a complex manifold. There is a $\mathbb{Z}$-bilinear commutative pairing

$$
\mathcal{B}(Y) \times \mathcal{B}(Y) \rightarrow \mathcal{B}(Y), \quad\left(\mu_{1}, \mu_{2}\right) \mapsto \mu_{1} \diamond_{Y} \mu_{2},
$$

with the following properties:

(i) $\mu_{1} \diamond_{Y} \mu_{2}$ has Zariski support on

$$
V:=\left|\mu_{1}\right| \cap\left|\mu_{2}\right| .
$$


(ii) For each $x \in Y$ we have

$$
\epsilon_{\ell}\left(\mu_{1}, \mu_{2}, x\right)=\operatorname{mult}_{x}\left(\mu_{1} \diamond_{Y} \mu_{2}\right)_{\ell}, \quad 0 \leq \ell \leq \operatorname{dim} V,
$$

where ()$_{\ell}$ denotes the component of dimension $\ell$.

(iii) If $\mu_{1}, \mu_{2}$ are cycles that intersect properly, then $\mu_{1} \diamond_{Y} \mu_{2}=\mu_{1} \cdot Y \mu_{2}$.

(iv) The natural image in the cohomology group $\widehat{H}^{*, *}(V)$ of the component $\left(\mu_{1} \diamond_{Y} \mu_{2}\right)_{\rho}$ of the expected dimension $\rho, c f$. (1.1), coincides with the image of the Chow class $\mu_{1} \cdot Y \mu_{2}$.

For the definitions of the cohomology groups $\widehat{H}^{*, *}(V)$, see Sect. 2. Part (ii) means that $\diamond_{Y}$ solves our representation problem. However, (iv) suggests that already the component of dimension $\rho$ is as 'big' as the Chow class in a cohomological sense. In particular, if $Y=\mathbb{P}^{n}$ this implies that

$$
\operatorname{deg}\left(\mu_{1} \diamond \mathbb{P}^{n} \mu_{2}\right)_{\rho}=\operatorname{deg}\left(\mu_{1} \cdot \mathbb{P}^{n} \mu_{2}\right),
$$

which by Bézout's equality and (1.2) is equal to $\operatorname{deg}\left(\mu_{1} \bullet \mu_{2}\right)$ if $\rho \geq 0$. In general, the degree of the full generalized cycle class is much larger. For effective generalized cycle classes, in particular for cycles, we have the estimate, see Sect. 4,

$$
\operatorname{deg}\left(\mu_{1} \diamond \mathbb{P}^{n} \mu_{2}\right) \leq 2^{\operatorname{dim} V-\rho} \operatorname{deg}\left(\mu_{1} \bullet \mu_{2}\right) .
$$

The constant, which is the best possible, blows up when the intersection is far from being proper, i.e., when $\operatorname{dim} V-\rho$ is large.

It is thus natural to look for an extension of the $\bullet$-product, in order to get a representation of the local intersection numbers that is not 'too big'. To do this we restrict to a projective manifold $Y$. Let $L \rightarrow Y$ be a very ample line bundle. By definition then there is an embedding $i: Y \rightarrow \mathbb{P}^{M}$ for some $M$ such that $L=i^{*} \mathcal{O}(1)_{\mathbb{P}^{M}}$. Given $\mu_{1}, \mu_{2} \in \mathcal{B}(Y)$ we can define a product $\mu_{1} \bullet L \mu_{2}$ such that

$$
i_{*}\left(\mu_{1} \bullet L \mu_{2}\right)=i_{*} \mu_{1} \bullet i_{*} \mu_{2} .
$$

For $\mu \in \mathcal{B}_{k}(Y)$ we define

$$
\operatorname{deg}_{L} \mu=\int_{Y} \mu \wedge c_{1}(L)^{k}
$$

and extend to general $\mu \in \mathcal{B}(Y)$ by linearity. Here is our second main result.

Theorem 1.2 Assume that $Y$ is a projective manifold and let $L \rightarrow Y$ be a very ample line bundle.

(i) The pairing $\mathcal{B}(Y) \times \mathcal{B}(Y) \rightarrow \mathcal{B}(Y),\left(\mu_{1}, \mu_{2}\right) \mapsto \mu_{1} \bullet_{L} \mu_{2}$, defined by (1.5) is commutative and $\mathbb{Z}$-bilinear. It depends on the choice of $L$ but not on the embedding $i$.

(ii) The product $\mu_{1} \bullet \mu_{2} \mu_{2}$ has Zariski support on $V=\left|\mu_{1}\right| \cap\left|\mu_{2}\right|$.

(iii) For each $x \in Y$ we have

$$
\operatorname{mult}_{x}\left(\mu_{1} \bullet L \mu_{2}\right)_{\ell}=\epsilon_{\ell}\left(\mu_{1}, \mu_{2}, x\right), \quad \ell=0,1, \ldots, \operatorname{dim} V,
$$

where ( ) $\ell$ denotes the component of dimension $\ell$.

(iv) If the $\mu_{1}$ and $\mu_{2}$ are effective, then $\mu_{1} \bullet L \mu_{2}$ is effective and

$$
\operatorname{deg}_{L}\left(\mu_{1} \bullet L \mu_{2}\right) \leq \operatorname{deg}_{L} \mu_{1} \cdot \operatorname{deg}_{L} \mu_{2} \text {. }
$$


(v) If $\mu_{1}, \mu_{2}$ are cycles that intersect properly, then

$$
\mu_{1} \bullet L \mu_{2}=\mu_{1} \cdot Y \mu_{2}+\cdots,
$$

where $\cdots$ are terms with lower dimension and vanishing multiplicities.

In view of (iii) thus $\mu_{1} \bullet_{L} \mu_{2}$ has the 'right' multiplicities at each point, whereas (iv) says that we have control of the total mass of $\mu_{1} \bullet L \mu_{2}$. In case $Y=\mathbb{P}^{n}$ and $L=\mathcal{O}(1)$, then $\bullet L$ coincides with $\bullet$. In this case the dots in (1.9) vanish. However, in general they do not, see Example 7.3 in Sect. 7.

The plan of this paper is as follows. In Sect. 2 we recall necessary material from [4,5]. The $\diamond_{Y}$-product is defined in Sect. 3 and Theorem 1.1 is proved. The relation to the $\bullet$-product on $\mathbb{P}^{n}$ is discussed in Sect. 4. In Sect. 5 we prove Theorem 1.2 and provide formulas that relate $\diamond_{Y}$ and $\bullet_{L}$. In Sect. 6 we provide some further properties of these products, and in the final section, Sect. 7, we give various explicit examples.

\section{Preliminaries}

Throughout this section $X$ is a reduced analytic space of dimension $n$. We let $\mathcal{Z}_{k}(X)$ denote the $\mathbb{Z}$-module of $k$-cycles on $X$. Given $\mu \in \mathcal{Z}_{k}(X)$ there is the associated closed current $[\mu]$, the Lelong current, of bidegree $(n-k, n-k)$. We will often identify $\mu$ and its Lelong current. If nothing else is stated the definitions and results in this section are from [4, Sections 3 and 4].

\subsection{Generalized cycles}

The group $\mathcal{G Z}_{k}(X)$ of generalized cycles of dimension $k$ was introduced in [4]. It is the $\mathbb{Z}$-module generated by (closed) $(n-k, n-k)$-currents of the form $\tau_{*} \alpha$, where $\tau: W \rightarrow X$ is a proper mapping and

$$
\alpha=\hat{c}_{1}\left(L_{1}\right) \wedge \ldots \wedge \hat{c}_{1}\left(L_{r}\right),
$$

where $L_{j} \rightarrow W$ are Hermitian ${ }^{1}$ line bundles, and $\hat{c}_{1}\left(L_{j}\right)$ are the associated first Chern forms. We let $\mathcal{G} \mathcal{Z}(X)=\oplus_{0}^{n} \mathcal{G Z}_{k}(X)$. Here $W$ can be any complex variety but by virtue of Hironaka's theorem we may assume that $W$ is a connected manifold. It is clear that generalized cycles are closed currents of order 0. Moreover, their Lelong numbers (multiplicities, see below) are integers. This means that $\mathcal{G} \mathcal{Z}(X)$ is a quite restricted class of closed currents. We are basically interested in a certain quotient space $\mathcal{B}(X)=\oplus_{0}^{n} \mathcal{B}_{k}(X)$, where $\mathcal{B}_{k}(X)$ are quotient spaces of $\mathcal{G Z}_{k}(X)$. For precise definitions and proofs of the properties listed below, see [4, Sections 3 and 4].

(i) We have a natural inclusion $\mathcal{Z}_{k}(X) \rightarrow \mathcal{B}_{k}(X)$ for each $k$ and hence an inclusion $\mathcal{Z}(X)=\oplus_{0}^{n} \mathcal{Z}_{k}(X) \rightarrow \mathcal{B}(X)=\oplus_{0}^{n} \mathcal{B}_{k}(X)$.

(ii) Each $\mu \in \mathcal{B}_{k}(X)$ has a well-defined Zariski support $|\mu|$; it is the smallest Zariski closed set such that $\mu$ has a representative in $\mathcal{G Z}_{k}(X)$ that vanishes in its complement.

(iii) Given $\mu \in \mathcal{B}(X)$ also its restriction $\mathbf{1}_{V} \mu$ to the subvariety $V \subset X$ is an element in $\mathcal{B}(X)$.

(iv) If $f: X \rightarrow X^{\prime}$ is a proper mapping, then the push-forward $f_{*}$ induces a mapping $f_{*}: \mathcal{B}_{k}(X) \rightarrow \mathcal{B}_{k}\left(X^{\prime}\right)$ that coincides with the usual push-forward on cycles.

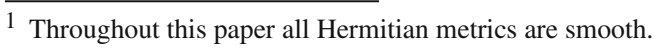


(v) If $i: X \rightarrow X^{\prime}$ is an embedding, then $i_{*}: \mathcal{B}_{k}(X) \rightarrow \mathcal{B}_{k}\left(X^{\prime}\right)$ is injective, and the image is precisely the elements in $\mathcal{B}_{k}\left(X^{\prime}\right)$ with Zariski support on $i(X)$.

(vi) If $E \rightarrow X$ is a vector bundle, then we have natural mappings $c_{k}(E): \mathcal{B}_{*}(X) \rightarrow$ $\mathcal{B}_{*-k}(X)$. The image of $\mu$ is represented by $\hat{c}_{k}(E) \wedge \hat{\mu}$, where $\hat{\mu} \in \mathcal{G} \mathcal{Z}(X)$ represents $\mu$ and $\hat{c}_{k}(E)$ is the Chern form associated with a (smooth) Hermitian metric on $E$.

(vii) If $f: X \rightarrow X^{\prime}$ is a proper mapping and $E^{\prime} \rightarrow X^{\prime}$ is a vector bundle, then $f^{*} c_{k}\left(E^{\prime}\right)=$ $c_{k}\left(f^{*} E^{\prime}\right)$, and if $\mu \in \mathcal{B}_{*}(X)$, then

$$
f_{*}\left(f^{*} c_{k}\left(E^{\prime}\right) \wedge \mu\right)=c_{k}\left(E^{\prime}\right) \wedge f_{*} \mu .
$$

(viii) If $\mu \in \mathcal{B}(X)$ and $\mu^{\prime} \in \mathcal{B}\left(X^{\prime}\right)$, where $X^{\prime}$ is another reduced analytic space, then there is a well-defined element $\mu \times \mu^{\prime} \in \mathcal{B}\left(X \times X^{\prime}\right)$, see [5, Lemma 2.1].

In the recent paper [14] Yger introduces the related notion of algebraic generalized cycle as a generalization of (complex) algebraic cycle.

\subsection{Irreducibility}

A generalized cycle class $\mu \in \mathcal{B}(X)$ is irreducible if its Zariski support $|\mu|$ is an irreducible subvariety and $\mu$ has a representative $\hat{\mu}$ with Zariski support $|\mu|$ such that $\mathbf{1}_{W} \hat{\mu}=0$ for each subvariety $W \subset X$ that does not contain $|\mu|$. This condition on $\hat{\mu}$ is equivalent to that $\hat{\mu}$ is a (finite) sum of elements of the form $\tau_{*} \alpha$, where $\alpha$ is a form as in (2.1) on $W$, and $\tau: W \rightarrow|\mu|$ is surjective. Notice that these various terms can have different dimensions.

Each element in $\mathcal{G} \mathcal{Z}(X)$, and in $\mathcal{B}(X)$, has a unique decomposition in irreducible components with different Zariski supports. Each irreducible element has in turn a unique decomposition in components of various dimensions.

There is a unique decomposition

$$
\mu=\mu_{\text {fix }}+\mu_{\text {mov }},
$$

where $\mu_{f i x}$ is an ordinary cycle, whose irreducible components are called the fixed components of $\mu$, and $\mu_{\text {mov }}$, whose irreducible components are the moving components. Each moving component has strictly lower dimension than its Zariski support.

\subsection{Multiplicities}

If $\mu$ is a cycle, then the multiplicity mult $x_{x} \mu$ at $x \in X$ is precisely the Lelong number at $x$ of the associated Lelong current. If $X$ is not smooth, then mult $_{x} \mu=$ mult $_{i(x)} i_{*} \mu$ if $i: X \rightarrow X^{\prime}$ is an embedding and $X^{\prime}$ is smooth. There is a suitable definition of Lelong number that extends to all generalized cycles and it turns out to depend only of their classes in $\mathcal{B}(X)$, see [4, Section 6]. In this way we have for each $\mu \in \mathcal{B}_{k}(X)$ well-defined multiplicities mult t $_{x} \mu$ at all points $x \in X$, and these numbers are integers. They are local in the following sense:

If $U \subset X$ is an open subset, then we have natural restriction mappings $r_{U}: \mathcal{B}_{k}(X) \rightarrow$ $\mathcal{B}_{k}(U)$, and mult $_{x} \mu=$ mult $_{x} r_{U} \mu$.

If $i: X \rightarrow X^{\prime}$, where $X^{\prime}$ is smooth and $\mu \in \mathcal{B}(X)$, then

$$
\operatorname{mult}_{x} \mu=\operatorname{mult}_{i(x)} i_{*} \mu \text {. }
$$

Assume that $\mu=\gamma \wedge \mu^{\prime}$, where $\mu, \mu^{\prime} \in \mathcal{B}(U)$ and $\gamma$ is smooth and has positive degree. Then mult $_{x} \mu=0$. 


\subsection{Effective generalized cycle classes}

In [5, Section 2.4] was introduced the notion of effective generalized cycle class $\mu \in \mathcal{B}(X)$ generalizing the notion of effective cycle. It means precisely that $\mu$ has a representative $\hat{\mu} \in \mathcal{G} \mathcal{Z}(X)$ that is a positive current. Effective generalized cycle classes have non-negative multiplicities at each point.

\subsection{The cohomology groups $\widehat{H}^{*, *}(X)$}

We define $\widehat{H}^{*, *}(X)$ as the vector space of closed $(*, *)$-currents of order 0 modulo the subspace generated by all $d \tau$ for currents $\tau$ of order 0 such that also $d \tau$ has order 0 , cf. [4, Section 10].

If $f: X \rightarrow X^{\prime}$ is proper and $n^{\prime}=\operatorname{dim} X^{\prime}$, then we have natural mappings $f_{*}: \widehat{H}^{n-*, n-*}(X) \rightarrow$ $\widehat{H}^{n^{\prime}-*, n^{\prime}-*}\left(X^{\prime}\right)$.

If $X$ is smooth, then $\widehat{H}^{*, *}(X)$ is naturally isomorphic to the usual cohomology groups $H^{*, *}(X, \mathbb{C})$.

For each $k$ there is a natural mapping $r_{k}: \mathcal{Z}_{k}(X) \rightarrow \widehat{H}^{n-k, n-k}(X)$ that takes $\mu \in \mathcal{Z}_{k}(X)$ to its Lelong current $[\mu]$. This mapping extends to a mapping $\mathcal{B}_{k}(X) \rightarrow \widehat{H}^{n-k, n-k}(X)$.

Each $\mu \in \mathcal{Z}_{k}(X)$ defines an element in the Chow group $\mathcal{A}_{k}(X)$ and the mapping $r_{k}$ induces a mapping $\mathcal{A}_{k}(X) \rightarrow \widehat{H}^{n-k, n-k}(X)$.

\subsection{The $\mathcal{B}$-Segre class}

Assume that $\mathcal{J}$ is a coherent ideal sheaf on $X$ with zero set $Z$. Also assume that $\mathcal{J}$ is generated by a holomorphic section $\sigma$ of a Hermitian vector bundle $E \rightarrow X$. That is, $\mathcal{J}$ is locally generated by the tuple of holomorphic functions obtained when $\sigma$ is expressed in a local frame of $E$. Such a section $\sigma$ exists if $X$ is projective. For any $\mu \in \mathcal{B}(X)$, following [4, Section 5], let

$$
M_{k}^{\sigma} \wedge \hat{\mu}=\mathbf{1}_{Z}\left(d d^{c} \log |\sigma|^{2}\right)^{k} \wedge \hat{\mu}:=\mathbf{1}_{Z} \lim _{\epsilon \rightarrow 0}\left(d d^{c} \log \left(|\sigma|^{2}+\epsilon\right)\right)^{k} \wedge \hat{\mu}, \quad k=0,1, \ldots,
$$

where $\hat{\mu}$ is a representative of the class $\mu$. The existence of the limit is highly non-trivial and relies on a resolution of singularities. Then $M_{k}^{\sigma} \wedge \hat{\mu}$ defines a class $S_{k}(\mathcal{J}, \mu)$ in $\mathcal{B}_{\ell}(X)$, $\ell=\operatorname{dim} \mu-k$, that only depends on $\mu$ and $\mathcal{J}$. Clearly $\mu \mapsto S_{k}(\mathcal{J}, \mu)$ is $\mathbb{Z}$-linear. Let $S(\mathcal{J}, \mu)=S_{0}(\mathcal{J}, \mu)+\cdots+S_{\operatorname{dim} \mu}(\mathcal{J}, \mu)$.

Let $M^{\sigma} \wedge \hat{\mu}=M_{0}^{\sigma} \wedge \hat{\mu}+\cdots+M_{\operatorname{dim} \hat{\mu}}^{\sigma} \wedge \hat{\mu}$. If $f: X \rightarrow X^{\prime}$ is proper, $\mathcal{J}$ is an ideal sheaf on $X^{\prime}$, and $\mu \in \mathcal{B}(X)$, then $f_{*}\left(M^{f^{*} \sigma} \wedge \hat{\mu}\right)=M^{\sigma} \wedge f_{*} \hat{\mu}$ and hence ${ }^{2}$

$$
f_{*} S\left(f^{*} \mathcal{J}, \mu\right)=S\left(\mathcal{J}, f_{*} \mu\right) .
$$

If $\mathcal{J}$ is locally a complete intersection, that is, defines a regular embedding, then one can define the Segre classes $S(\mathcal{J}, \mu)$ without a section $\sigma$ as above. We show this in Sect. 3 below in the case that $\mathcal{J}$ is the ideal sheaf of a submanifold $V \subset X$.

${ }^{2}$ In this paper $f^{*} \mathcal{J}$ denotes the sheaf over $X$ generated by pullbacks of sections of $\mathcal{J}$. 


\subsection{Segre numbers}

Given a coherent ideal sheaf $\mathcal{J} \rightarrow X$ with zero set $Z$, and $\mu \in \mathcal{B}(X)$, there are, at each point $x$, non-negative integers $e_{k}(\mathcal{J}, X, x)$ for $k=0,1, \ldots, \operatorname{dim} Z$, called the Segre numbers. They were introduced independently by Tworzewski, [12], and Gaffney-Gassler, [8], as the multiplicity of the component of codimension $k$ of a generic local SV-cycle in $\mathcal{J}_{x}$. A purely algebraic definition was introduced in [1] and the equivalence to the geometric definition was proved in [2]. If $Z$ is a point, then the Segre number is precisely the Hilbert-Samuel multiplicity. In [3] was introduced an analytic definition.

Given $\mu \in \mathcal{B}(X)$ we have the integers

$$
e_{k}(\mathcal{J}, \mu, x):=\operatorname{mult}_{x} S_{k}(\mathcal{J}, \mu),
$$

that are called the Segre numbers of $\mathcal{J}$ on $\mu$ in [4, Section 2.6]. If $\sigma$ is a section of a Hermitian vector bundle that defines $\mathcal{J}$ and $\hat{\mu}$ is a representative of $\mu$, then

$$
e_{k}(\mathcal{J}, \mu, x)=\operatorname{mult}_{x} M_{k}^{\sigma} \wedge \hat{\mu} .
$$

Locally we can choose $\sigma$ and the (smooth) Hermitian metric so that $\log |\sigma|^{2}$ is plurisubharmonic. If follows from (2.8) and (2.5), and the Skoda-El Mir theorem, that the Segre numbers $e_{k}(\mathcal{J}, \mu, x)$ are non-negative if $\mu$ is effective. We have that $e_{k}(\mathcal{J}, X, x)=e_{k}\left(\mathcal{J}, \mathbf{1}_{X}, x\right)$, see [4].

\subsection{Local intersection numbers}

Let $X$ be smooth, assume that $\mu_{1}, \mu_{2} \in \mathcal{B}(X)$ have pure dimensions, and let $d=\operatorname{dim} \mu_{1}+$ $\operatorname{dim} \mu_{2}$. Furthermore, let $\mathcal{J}_{\Delta}$ be the sheaf that defines the diagonal $\Delta$ in $X \times X$ and let $j: X \rightarrow X \times X$ be the natural parametrization. We define the local intersection numbers

$$
\epsilon_{\ell}\left(\mu_{1}, \mu_{2}, x\right)=e_{d-\ell}\left(\mathcal{J}_{\Delta}, \mu_{1} \times \mu_{2}, j(x)\right), \quad \ell=0,1, \ldots,
$$

saying that $\epsilon_{\ell}\left(\mu_{1}, \mu_{2}, x\right)$ is the local intersection number at dimension $\ell$. These numbers are biholomorphic invariants, and if we have an embedding $i: X \rightarrow X^{\prime}$ in a larger manifold $X^{\prime}$, then it follows from (2.6), (2.7) and (2.9) that

$$
\epsilon_{\ell}\left(\mu_{1}, \mu_{2}, x\right)=\epsilon_{\ell}\left(i_{*} \mu_{1}, i_{*} \mu, i(x)\right)
$$

for each $x \in X$.

\section{The $\diamond y$-product}

Let $Y$ be a complex manifold, let $j: Y \rightarrow Y \times Y$ be the natural parametrization of the diagonal $\Delta$ in $Y \times Y$, and let $\mathcal{J}_{\Delta}$ be the corresponding ideal sheaf. To define the $\diamond_{Y}$-product $\mu_{1} \diamond_{Y} \mu_{2}$ for $\mu_{1}, \mu_{2} \in \mathcal{B}(Y)$ we need a definition of $S\left(\mathcal{J}_{\Delta}, \mu_{1} \times \mu_{2}\right)$ when $\mathcal{J}_{\Delta}$ is not necessarily generated by a global holomorphic section of a Hermitian vector bundle $E \rightarrow Y \times Y$.

Let $X$ be a complex manifold, $i: V \rightarrow X$ a submanifold, and $\mathcal{J}_{V}$ the corresponding ideal sheaf. Recall that if there is a holomorphic section $\sigma$ of a vector bundle $E \rightarrow X$ such that $\sigma$ generates $\mathcal{J}_{V}$, then there is an embedding $\left.N_{V} X \hookrightarrow E\right|_{V}$; see, e.g. [4, Lemma 7.3].

Proposition 3.1 Assume that the normal bundle $N_{V} X \rightarrow V$ is equipped with a Hermitian metric. For any $\hat{\mu} \in \mathcal{G} \mathcal{Z}(X)$ and $k=0,1,2, \ldots$, there is a generalized cycle $\widehat{S}_{k}\left(\mathcal{J}_{V}, \hat{\mu}\right) \in$ $\mathcal{G Z}_{\operatorname{dim} \hat{\mu}-k}(X)$ with the following properties. 
(i) If $U \subset X$ is open and $\sigma$ is a holomorphic section of a Hermitian vector bundle $E \rightarrow U$ such that $\sigma$ generates $\mathcal{J}_{V}$ in $U$ and the embedding $\left.N_{V} U \hookrightarrow E\right|_{V \cap U}$ is an embedding of Hermitian vector bundles, then $\widehat{S}_{k}\left(\mathcal{J}_{V}, \hat{\mu}\right)=M_{k}^{\sigma} \wedge \hat{\mu}$ in $U$.

(ii) The image of $\widehat{S}_{k}\left(\mathcal{J}_{V}, \hat{\mu}\right)$ in $\mathcal{B}_{\operatorname{dim} \hat{\mu}-k}(X)$ only depends on the image of $\hat{\mu}$ in $\mathcal{B}(X)$; in particular it is independent of the Hermitian metric on $N_{V} X \rightarrow V$.

If $\mu \in \mathcal{B}(X)$ we let $S_{k}\left(\mathcal{J}_{V}, \mu\right)$ be the image in $\mathcal{B}_{\operatorname{dim} \mu-k}(X)$ of $\widehat{S}_{k}\left(\mathcal{J}_{V}, \hat{\mu}\right)$, where $\hat{\mu} \in$ $\mathcal{G} \mathcal{Z}(X)$ is any representative of $\mu$.

Proof The proof is based on some ideas in [10]. Let $\hat{\mu} \in \mathcal{G Z}(X)$ and assume that $\hat{\mu}=\tau_{*} \alpha$, where $\tau: W \rightarrow X$ is a proper holomorphic mapping and $\alpha$ is a product of first Chern forms of Hermitian line bundles on $W$. We can assume that $\tau^{*} \mathcal{J}_{V}$ is principal and that $W$ is smooth. Consider the commutative diagram

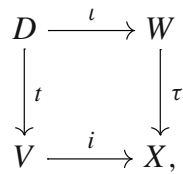

where $D$ is the divisor of $\tau^{*} \mathcal{J}_{V}$. Let $L \rightarrow W$ be the line bundle corresponding to $D$; for future reference we recall that $\left.L\right|_{D}$ is the normal bundle of $D$. We will show below that the Hermitian metric on $N_{V} X$ induces a metric on $\left.L\right|_{D}$. Let $\omega=\hat{c}_{1}\left(\left.L\right|_{D} ^{*}\right)$ be the first Chern form of the dual bundle. Then

$$
\tau_{*}\left([D] \wedge \omega^{k-1} \wedge \alpha\right)=i_{*} t_{*} \iota^{*}\left(\omega^{k-1} \wedge \alpha\right)
$$

is in $\mathcal{G} \mathcal{Z}(X)$. This will be our definition of $\widehat{S}_{k}\left(\mathcal{J}_{V}, \hat{\mu}\right)$. However, a priori this definition depends on the representation $\tau_{*} \alpha$ of $\hat{\mu}$.

Let us now describe the induced metric on $\left.L\right|_{D}$. Let $\kappa=\operatorname{codim} V$. We recall the following ad hoc definition; cf. [4, Section 7]. A section $\xi$ of $N_{V} X$ is a choice of a $\kappa$-tuple $\xi(s)$ locally on $V$ for each local holomorphic $\kappa$-tuple $s$ generating $\mathcal{J}_{V}$ such that $\xi(M s)=M \xi(s)$ on $V$ for any locally defined holomorphic matrix $M$ invertible in a neighborhood of $V$.

Assume that $U, \sigma$, and $E$ are as in (i). Assume also that $s$ is a holomorphic $\kappa$-tuple generating $\mathcal{J}_{V}$ in an open set $U^{\prime}$. In view of the definition of a section of $N_{V} X$ above, if we consider $s$ as a section of the trivial rank $\kappa$ bundle $F \rightarrow U^{\prime}$, then we can identify $\left.F\right|_{V \cap U^{\prime}}$ with $N_{V} U^{\prime}$. Notice that this identification induces a Hermitian metric on $\left.F\right|_{V \cap U^{\prime}}$; we extend it to a Hermitian metric on $F$ in an arbitrary way. Since both $s$ and $\sigma$ generate $\mathcal{J}_{V}$ in $U \cap U^{\prime}$ there is a holomorphic $A \in \operatorname{Hom}(F, E)$ in $U \cap U^{\prime}$ such that $\sigma=A s$. In $V \cap U \cap U^{\prime}$, the embedding $\left.N_{V} U \hookrightarrow E\right|_{V \cap U}$, which by assumption is an embedding of Hermitian bundles, is then realized by $\left.A\right|_{V} ;$ cf. [4, Lemma 7.3].

Let $U^{\prime \prime}=\tau^{-1}\left(U \cap U^{\prime}\right)$. In $D \cap U^{\prime \prime}$ we get that $a:=\left.\tau^{*} A\right|_{D \cap U^{\prime \prime}}$ embeds $\tau^{*} N_{V} U$ in $\left.\tau^{*} E\right|_{D}$. Moreover, we have a similar situation in $\tau^{-1}\left(U^{\prime}\right)$ and in $\tau^{-1}(U)$ as we had in $U \cap U^{\prime}$ since the ideal sheaf $\tau^{*} \mathcal{J}_{V}$, which defines $D$, is generated by $\tau^{*} s$ in $\tau^{-1}\left(U^{\prime}\right)$ and $\tau^{*} \sigma$ in $\tau^{-1}(U)$. In the same way as above, since $\left.L\right|_{D}$ is the normal bundle of $D$, we thus get embeddings

$$
\left.\left.L\right|_{D \cap \tau^{-1}\left(U^{\prime}\right)} \hookrightarrow \tau^{*} F\right|_{D \cap \tau^{-1}\left(U^{\prime}\right)}=\tau^{*} N_{V} U^{\prime} \text { and }\left.\left.L\right|_{D \cap \tau^{-1}(U)} \hookrightarrow \tau^{*} E\right|_{D \cap \tau^{-1}(U)} .
$$

Using the ad hoc definition of a section of a normal bundle it is straightforward to check that the latter embedding restricted to $U^{\prime \prime}$ is the composition of

$$
\left.\left.\left.L\right|_{D \cap U^{\prime \prime}} \hookrightarrow \tau^{*} F\right|_{D \cap U^{\prime \prime}} \stackrel{a}{\hookrightarrow} \tau^{*} E\right|_{D \cap U^{\prime \prime}}
$$


It follows that the metrics induced on $\left.L\right|_{D}$ by the embeddings in $\tau^{*} F$ and $\tau^{*} E$, respectively, coincide on $\left.L\right|_{D \cap U^{\prime \prime}}$. In particular, if $\sigma$ is a holomorphic $\kappa$-tuple generating $\mathcal{J}_{V}$ in $U$, so that $\left.E\right|_{V \cap U}$ can be identified with $N_{V} U$, it follows that the metric on $N_{V} X$ induces a metric on $\left.L\right|_{D}$.

With this metric on $\left.L\right|_{D}, M_{k}^{\sigma} \wedge \hat{\mu}$ equals the left-hand side of (3.1) in $U$ by [4, Eq. (5.9)]. In view of (2.5), $M_{k}^{\sigma} \wedge \hat{\mu}$ is independent of the representation $\tau_{*} \alpha$ of $\hat{\mu}$. It follows that (3.1) is independent of the representation $\tau_{*} \alpha$ of $\hat{\mu}$, and we take (3.1) as our definition of $\widehat{S}_{k}\left(\mathcal{J}_{V}, \hat{\mu}\right)$. Then $M_{k}^{\sigma} \wedge \hat{\mu}=\widehat{S}_{k}\left(\mathcal{J}_{V}, \hat{\mu}\right)$ in $U$ and (i) is proved.

We now note that (ii) follows. Indeed, in view of [4, Section 3], the image of (3.1) in $\mathcal{B}(X)$ is 0 if $\hat{\mu}$ is 0 in $\mathcal{B}(X)$ and, moreover, it is independent of the Hermitian metric on $L$.

Let $N_{\Delta}(Y \times Y) \rightarrow \Delta$ be the normal bundle.

Definition 3.2 Given $\mu_{1}, \mu_{2} \in \mathcal{B}(Y), \mu_{1} \diamond_{Y} \mu_{2}$ is the unique element in $\mathcal{B}(Y)$ such that

$$
j_{*}\left(\mu_{1} \diamond_{Y} \mu_{2}\right)=c\left(N_{\Delta}(Y \times Y)\right) \wedge S\left(\mathcal{J}_{\Delta}, \mu_{1} \times \mu_{2}\right) .
$$

If we identify $\Delta$ and $Y$, then $N_{\Delta}(Y \times Y)$ is isomorphic to $T Y$ so if we identify $S\left(\mathcal{J}_{\Delta}, \mu_{1} \times\right.$ $\mu_{2}$ ) with an element in $\mathcal{B}(Y)$, then

$$
\mu_{1} \diamond_{Y} \mu_{2}=c(T Y) \wedge S\left(\mathcal{J}_{\Delta}, \mu_{1} \times \mu_{2}\right) .
$$

For the proof of Theorem 1.1 we need the following lemma. Recall that a coherent ideal sheaf $\mathcal{J} \rightarrow X$, with zero set $Z$, on a reduced space $X$ of pure dimension defines a regular embedding of codimension $\kappa$ if $\operatorname{codim} Z=\kappa$ and locally $\mathcal{J}$ is generated by $\kappa$ functions. Then there is a well-defined normal bundle $N_{\mathcal{J}} X$ over $Z$. See, e.g., [4, Section 7].

Lemma 3.3 Let $X^{\prime}$ be a reduced space and let $\iota: X \rightarrow X^{\prime}$ be a reduced subspace. Assume that the coherent sheaf $\mathcal{J}^{\prime} \rightarrow X^{\prime}$ defines a regular embedding of codimension $\kappa$ in $X^{\prime}$, and that $\mathcal{J}=\iota^{*} \mathcal{J}^{\prime}$ defines a regular embedding of codimension $\kappa$ in $X$. Then $N_{\mathcal{J}} X=\iota^{*} N_{\mathcal{J}^{\prime}} X^{\prime}$.

Let $Z$ and $Z^{\prime}$ denote the zero sets of $\mathcal{J}$ and $\mathcal{J}^{\prime}$, respectively.

Proof By assumption, locally we have a set of generators $s=\left(s_{1}, \ldots, s_{\kappa}\right)$ for $\mathcal{J}^{\prime}$. If $s^{\prime}$ is another such $\kappa$-tuple, then (on the overlap) there is an invertible holomorphic $\kappa \times \kappa$ matrix $a\left(s, s^{\prime}\right)$ such that $s^{\prime}=a\left(s, s^{\prime}\right) s$. The matrices so obtained form the transition matrices on $Z^{\prime}$ for the bundle $N_{\mathcal{J}^{\prime}} X^{\prime}$. Now the lemma follows by noting that $\iota^{*} s$ and $\iota^{*} s^{\prime}$ are minimal sets of generators for $\mathcal{J}=\iota^{*} \mathcal{J}^{\prime}$ and hence $\iota^{*} a\left(s, s^{\prime}\right)$ are transition matrices for $N_{\mathcal{J}} X \rightarrow Z$.

Proof of Theorem 1.1 It is clear that $\mu_{1} \diamond_{Y} \mu_{2}$ is $\mathbb{Z}$-bilinear and commutative since $S\left(\mathcal{J}_{\Delta}, \mu_{1} \times\right.$ $\left.\mu_{2}\right)$ is, cf. Sect. 2.6. Moreover, its Zariski support is contained in $\Delta \cap\left(\left|\mu_{1}\right| \times\left|\mu_{2}\right|\right)$ which after identifying $\Delta$ and $Y$ is precisely $V=\left|\mu_{1}\right| \cap\left|\mu_{2}\right|$. Thus (i) holds.

It follows from (2.7) and (2.9) that

$$
\epsilon_{\ell}\left(\mu_{1}, \mu_{2}, x\right)=\operatorname{mult}_{j(x)} S_{k}\left(\mathcal{J}_{\Delta}, \mu_{1} \times \mu_{2}\right),
$$

where $k=\operatorname{dim} \mu_{1}+\operatorname{dim} \mu_{2}-\ell$. Since $c\left(N_{\Delta}(Y \times Y)\right)=1+\cdots$, where $\cdots$ are smooth forms of positive bidegree, it follows from the dimension principle that

$$
j_{*}\left(\mu_{1} \diamond_{Y} \mu_{2}\right)_{\ell}=\left(c\left(N_{\Delta}(Y \times Y)\right) \wedge S\left(\mathcal{J}_{\Delta}, \mu_{1} \times \mu_{2}\right)\right)_{\ell}=S_{k}\left(\mathcal{J}_{\Delta}, \mu_{1} \times \mu_{2}\right)+\cdots,
$$

where $\cdots$ are smooth forms of positive degree times generalized cycle classes. Now (ii) follows from (3.3), (3.4) and the comment after (2.4). 
We now prove (iii). Assume that $\mu_{j}$ are cycles that intersect properly. Then $\Delta$ intersects $X:=\mu_{1} \times \mu_{2}$ properly so that if $\iota: X \rightarrow Y \times Y$, then $\mathcal{J}:=\iota^{*} \mathcal{J}_{\Delta}$ defines a regular embedding in $X$. In view of Lemma 3.3, (2.6) and (2.2) we have, using the notation $S\left(\mathcal{J}, \mathbf{1}_{X}\right)=S(\mathcal{J}, X)$,

$$
j_{*}\left(\mu_{1} \diamond_{Y} \mu_{2}\right)=c\left(N_{\mathcal{J}_{\Delta}}(Y \times Y)\right) \wedge S\left(\mathcal{J}_{\Delta}, X\right)=\iota_{*}\left(c\left(N_{\mathcal{J}} X\right) \wedge S(\mathcal{J}, X)\right)=\iota_{*}\left[Z_{\mathcal{J}}\right],
$$

where the last equality is precisely [4, Theorem 1.4]. Here $\left[Z_{\mathcal{J}}\right]$ is the Lelong current of the fundamental cycle associated with $\mathcal{J}$. Its Zariski support is precisely $Z$ but there is a certain multiplicity of each irreducible component of $Z$. Since the right hand side of (3.5) has the expected dimension $\rho$, cf. (1.1), (3.5) implies that

$$
j_{*}\left(\mu_{1} \diamond_{Y} \mu_{2}\right)_{\rho}=\iota_{*}\left[Z_{\mathcal{J}}\right] .
$$

Furthermore, $\left(\mu_{1} \diamond_{Y} \mu_{2}\right)_{\rho}=\mu_{1} \cdot \mathcal{B}(Y) \mu_{2}$, where $\mu_{1} \cdot \mathcal{B}(Y) \mu_{2}$ is the product from [5, Section 5]. Since $\mu_{j}$ intersect properly, this product is equal to $\mu_{1} \cdot{ }_{Y} \mu_{2}$ by [5, Proposition 5.8 (i)]. Thus

$$
\mu_{1} \cdot Y \mu_{2}=\left(\mu_{1} \diamond_{Y} \mu_{2}\right)_{\rho} .
$$

Now (iii) follows from (3.5)-(3.7).

We know from [5, Theorem 1.3] that the image of $\mu_{1} \cdot \mathcal{B}(Y) \mu_{2}$ coincides with the image of the Chow class $\mu_{1} \cdot Y \mu_{2}$. Thus (iv) follows from (3.7). This concludes the proof.

For future reference we include the following simple proposition.

Proposition 3.4 Assume that we have an embedding $i: Y \rightarrow Y^{\prime}$ where $Y, Y^{\prime}$ are smooth. Then

$$
i_{*} \mu_{1} \diamond_{Y^{\prime}} i_{*} \mu_{2}=i_{*}\left(i^{*} c\left(N_{i(Y)} Y^{\prime}\right) \wedge \mu_{1} \diamond_{Y} \mu_{2}\right) .
$$

Proof Notice now that if $\Delta$ and $\Delta^{\prime}$ are the diagonals in $Y \times Y$ and $Y^{\prime} \times Y^{\prime}$, respectively, then

$$
\mathcal{J}_{\Delta}=(i \times i)^{*} \mathcal{J}_{\Delta^{\prime}} .
$$

We claim that

$$
(i \times i)_{*} S\left(\mathcal{J}_{\Delta}, \mu_{1} \times \mu_{2}\right)=S\left(\mathcal{J}_{\Delta^{\prime}}, i_{*} \mu_{1} \times i_{*} \mu_{2}\right) .
$$

If $\mathcal{J}_{\Delta^{\prime}}$ is generated by a holomorphic section of a Hermitian vector bundle over $Y^{\prime} \times Y^{\prime}$, then this follows from (2.6) since $(i \times i)_{*}\left(\mu_{1} \times \mu_{2}\right)=i_{*} \mu_{1} \times i_{*} \mu_{2}$. The general case follows since (2.6), with $f$ replaced by $i \times i, \mathcal{J}=\mathcal{J}_{\Delta^{\prime}}$, and $\mu=\mu_{1} \times \mu_{2}$, still holds in view of Proposition 3.1 .

Identifying $\mathcal{B}(\Delta)$ and $\mathcal{B}\left(\Delta^{\prime}\right)$ with $\mathcal{B}(Y)$ and $\mathcal{B}\left(Y^{\prime}\right)$, respectively, we have

$$
i_{*} S\left(\mathcal{J}_{\Delta}, \mu_{1} \times \mu_{2}\right)=S\left(\mathcal{J}_{\Delta^{\prime}}, i_{*} \mu_{1} \times i_{*} \mu_{2}\right) .
$$

By (3.2),

$$
S\left(\mathcal{J}_{\Delta}, \mu_{1} \times \mu_{2}\right)=c(T Y)^{-1} \wedge \mu_{1} \diamond_{Y} \mu_{2},
$$

and multiplying by $i^{*} c\left(T Y^{\prime}\right)=c(T Y) \wedge i^{*} c\left(N_{i(Y)} Y^{\prime}\right)$ we get

$$
i^{*} c\left(T Y^{\prime}\right) \wedge S\left(\mathcal{J}_{\Delta}, \mu_{1} \times \mu_{2}\right)=i^{*} c\left(N_{i(Y)} Y^{\prime}\right) \wedge \mu_{1} \diamond_{Y} \mu_{2} .
$$

Therefore, by (3.2), (3.10), and (3.11),

$$
\begin{aligned}
i_{*} \mu_{1} \diamond_{Y^{\prime}} i_{*} \mu_{2} & =c\left(T Y^{\prime}\right) \wedge S\left(\mathcal{J}_{\Delta^{\prime}}, i_{*} \mu_{1} \times i_{*} \mu_{2}\right)=i_{*}\left(i^{*}\left(c\left(T Y^{\prime}\right) \wedge S\left(\mathcal{J}_{\Delta}, \mu_{1} \times \mu_{2}\right)\right)\right. \\
& =i_{*}\left(i^{*} c\left(N_{i(Y)} Y^{\prime}\right) \wedge \mu_{1} \diamond_{Y} \mu_{2}\right) .
\end{aligned}
$$




\section{The $\diamond$ and $\bullet-$ products on $\mathbb{P}^{n}$}

We first recall the definition of the $\bullet$-product on $\mathbb{P}^{n}$. Let $\eta_{0}, \ldots, \eta_{n}$ be sections of $L=$ $\mathcal{O}(1)_{\mathbb{P}^{2 n+1}}$ that define the join diagonal $\Delta_{J}$ in $\mathbb{P}^{2 n+1}$, cf. [5, Section 6]. Let $\mathcal{J}_{J}$ be the sheaf that defines $\Delta_{J}$. Let $\eta_{k}^{\prime}$ be holomorphic functions that represent $\eta_{k}$ in a given local frame for $L$. Then

$$
d d^{c} \log |\eta|_{0}^{2}:=d d^{c} \log \left(\left|\eta_{0}^{\prime}\right|^{2}+\cdots+\left|\eta_{n}^{\prime}\right|^{2}\right)
$$

is a well-defined global current. For $\mu \in \mathcal{B}\left(\mathbb{P}^{2 n+1}\right)$ we define $V_{k}\left(\Delta_{J}, L, \mu\right)$ as the classes in $\mathcal{B}\left(\mathbb{P}^{2 n+1}\right)$ defined by

$$
M_{k}^{L, \eta} \wedge \hat{\mu}:=\mathbf{1}_{\Delta_{J}}\left(d d^{c} \log |\eta|_{\circ}^{2}\right)^{k} \wedge \hat{\mu}, \quad k=0, \ldots, n+1,
$$

where $\hat{\mu}$ is a generalized cycle that represents $\mu$. It is proved in [5, Section 4] that the Monge-Ampère products in (4.1) are well-defined and that $V_{k}\left(\Delta_{J}, L, \mu\right)$ is independent of the choice of representative $\hat{\mu}$ and sections $\eta_{1}, \ldots \eta_{n}$ defining $\Delta_{J}$. If $k>n+1$ in (4.1), then $V_{k}\left(\Delta_{J}, L, \mu\right)=0$. Let $V\left(\Delta_{J}, L, \mu\right)=V_{1}\left(\Delta_{J}, L, \mu\right)+V_{2}\left(\Delta_{J}, L, \mu\right)+\cdots$.

If $\mu_{1}, \mu_{2} \in \mathcal{B}\left(\mathbb{P}^{n}\right)$, then there is a natural class $\mu_{1} \times_{J} \mu_{2}$, see [5, Section 6], in $\mathcal{B}\left(\mathbb{P}^{2 n+1}\right)$, generalizing the usual join when $\mu_{1}, \mu_{2}$ are cycles, and $\operatorname{dim}\left(\mu_{1} \times_{J} \mu_{2}\right)=\operatorname{dim} \mu_{1}+\operatorname{dim} \mu_{2}+1$ if $\mu_{1}$ and $\mu_{2}$ have pure dimensions. Let $j: \mathbb{P}^{n} \rightarrow \mathbb{P}^{2 n+1},[x] \mapsto([x],[x])$, be the natural parametrization of $\Delta_{J}$.

For $\mu_{1}, \mu_{2} \in \mathcal{B}\left(\mathbb{P}^{n}\right)$ of pure dimensions

$$
\mu_{1} \bullet \mu_{2}=\sum_{\ell=0}^{n}\left(\mu_{1} \bullet \mu_{2}\right)_{\ell}
$$

is the class in $\mathcal{B}\left(\mathbb{P}^{n}\right)$ defined by

$$
j_{*}\left(\mu_{1} \bullet \mu_{2}\right)_{\ell}=V_{k(\ell)}\left(\Delta_{J}, L, \mu_{1} \times_{J} \mu_{2}\right),
$$

where

$$
k(\ell)=\operatorname{dim} \mu_{1}+\operatorname{dim} \mu_{2}+1-\ell .
$$

Let $\omega_{\mathbb{P}^{n}}$ be the first Chern class of $\mathcal{O}(1) \rightarrow \mathbb{P}^{n}$, for instance represented by the FubiniStudy metric form. If $i: W \rightarrow \mathbb{P}^{n}$ is a linear subspace, with the induced metric, then $\omega_{W}=$ $i^{*} \omega \mathbb{P}^{n}$. We will often write $\omega$ without subscript. Recall that $\hat{c}\left(T \mathbb{P}^{n}\right)=(1+\omega)^{n+1}$.

Proposition 4.1 Let $\diamond=\diamond_{\mathbb{P}^{n}}$ and let

$$
\rho=\operatorname{dim} \mu_{1}+\operatorname{dim} \mu_{2}-n, \quad V=\left|\mu_{1}\right| \cap\left|\mu_{2}\right| .
$$

We have the relations

$$
\mu_{1} \diamond \mu_{2}=\sum_{\ell=\max (0, \rho)}^{\operatorname{dim} V}(1+\omega)^{\ell-\rho}\left(\mu_{1} \bullet \mu_{2}\right)_{\ell} .
$$

and

$$
\mu_{1} \bullet \mu_{2}=\sum_{k=0}^{\operatorname{dim} V}(1-\omega)^{k-\rho}\left(\mu_{1} \diamond \mu_{2}\right)_{k} .
$$

Since $k \leq n+1, \ell \geq \rho$. Moreover, each term in the sum (4.2) has support on $V$. Hence the sum runs from $\max (\rho, 0)$ to $\operatorname{dim} V$. 
Proof With the notation in [5, Section 7] we have that $\mu_{1} \diamond \mu_{2}=i ! !\left(\mu_{1} \times \mu_{2}\right)$. It follows from [5, Proposition 7.1] that

$$
j_{*}\left(\mu_{1} \diamond \mu_{2}\right)=c\left(N_{\mathcal{J}_{J}} \mathbb{P}^{2 n+1}\right) \wedge S\left(\mathcal{J}_{J}, \mu_{1} \times_{J} \mu_{2}\right)=(1+\omega)^{n+1} \wedge S\left(\mathcal{J}_{J}, \mu_{1} \times_{J} \mu_{2}\right) .
$$

By the second van Gastel type equality in [4, Corollary 9.9] we get

$$
j_{*}\left(\mu_{1} \diamond \mu_{2}\right)=\sum_{k \geq 0}(1+\omega)^{n+1-k} \wedge V_{k}\left(\Delta_{J}, L, \mu_{1} \times_{J} \mu_{2}\right) .
$$

Since $k \leq n+1$ it follows that $\ell \geq \rho$, cf. (4.4), and since all terms in the sum have Zariski support on $V$, each term with $\ell$ larger than $\operatorname{dim} V$ must vanish in view of the dimension principle. Hence (4.7) is precisely (4.5).

The equality (4.6) follows from (4.5) and Lemma 4.2 below. Notice that although the sum in (4.5) happens to begin at $\ell=\max (\rho, 0)$ it will give rise to terms of lower dimension so (4.6) must start at $k=0$.

Lemma 4.2 Assume that $A=\sum_{\ell>0} A_{\ell}$ is a graded $\mathbb{C}$-algebra and $\omega: A \rightarrow$ A maps $A_{\ell+1} \rightarrow$ $A_{\ell}, \ell \geq 0$, and $A_{0} \rightarrow 0$. Moreover, let $r$ be a fixed integer. Assume that $a=a_{0}+a_{1}+\cdots$, where $a_{\ell}$ are elements in $A_{\ell}$, and let $b_{k}$ be the elements in $A_{k}$ so that

$$
\sum_{k=0}^{m} b_{k}=\sum_{\ell=0}^{m}(1+\omega)^{\ell+r} a_{\ell} .
$$

Then

$$
\sum_{\ell=0}^{m} a_{\ell}=\sum_{k=0}^{m}(1-\omega)^{k+r} b_{k} .
$$

This lemma is probably well-known but we sketch a proof.

Sketch of proof We can identify $a \in A$ with the $A$-valued meromorphic function

$$
z \mapsto \hat{a}(z)=\sum_{\ell \geq 0} z^{\ell+r} a_{\ell}
$$

Let

$$
T_{\omega} a:=\sum_{\ell=0}^{m}(1+\omega)^{\ell+r} a_{\ell} .
$$

Since $(z+\omega)^{\ell+r} a_{\ell}=(1+\omega / z)^{\ell+r} z^{\ell+r} a_{\ell}$ it follows that

$$
\widehat{T_{\omega} a}(z)=\sum_{\ell=0}^{m}(z+\omega)^{\ell+r} a_{\ell},
$$

i.e., $\widehat{T_{\omega} a}(z)$ is obtained by formally replacing each occurrence of $z$ in $\hat{a}(z)$ by $z+\omega$. It is now clear that $T_{-\omega} \circ T_{\omega}=I d$ which proves the lemma.

Recall, cf. (1.6), that $\mu \in \mathcal{B}_{k}\left(\mathbb{P}^{n}\right)$ has the degree

$$
\operatorname{deg} \mu:=\int_{\mathbb{P}^{n}} \omega^{k} \wedge \mu \text {. }
$$

If $\mu=\mu_{0}+\mu_{1}+\cdots$, where $\mu_{k}$ has pure dimension $k$, then $\operatorname{deg} \mu:=\operatorname{deg} \mu_{0}+\operatorname{deg} \mu_{1}+\cdots$. 
Proof of (1.4) From (4.5) we have that

$$
\mu_{1} \diamond \mu_{2}=\sum_{\ell=\max (0, \rho)}^{\operatorname{dim} V}(1+\omega)^{\ell-\rho}\left(\mu_{1} \bullet \mu_{2}\right)_{\ell}=\sum_{\ell=\max (0, \rho)}^{\operatorname{dim} V} \sum_{j=0}^{\min (\ell-\rho, \ell)}\left(\begin{array}{c}
\ell-\rho \\
j
\end{array}\right) \omega^{j} \wedge\left(\mu_{1} \bullet \mu_{2}\right)_{\ell}
$$

since $\omega^{j} \wedge\left(\mu_{1} \bullet \mu_{2}\right)_{\ell}=0$ for degree reasons when $j>\ell$. We get the estimate

$$
\begin{aligned}
\operatorname{deg}\left(\mu_{1} \diamond \mu_{2}\right) & =\sum_{\ell=\max (0, \rho)}^{\operatorname{dim} V} \sum_{j=0}^{\min (\ell-\rho, \ell)}\left(\begin{array}{c}
\ell-\rho \\
j
\end{array}\right) \operatorname{deg}\left(\omega^{j} \wedge\left(\mu_{1} \bullet \mu_{2}\right)_{\ell}\right) \\
& \leq \sum_{\ell=\max (0, \rho)}^{\operatorname{dim} V} \sum_{j=0}^{\ell-\rho}\left(\begin{array}{c}
\ell-\rho \\
j
\end{array}\right) \int_{\mathbb{P}^{n}} \omega^{\ell} \wedge\left(\mu_{1} \bullet \mu_{2}\right)_{\ell} \\
& =\sum_{\ell=\max (0, \rho)}^{\operatorname{dim} V} 2^{\ell-\rho} \int_{\mathbb{P}^{n}} \omega^{\ell} \wedge\left(\mu_{1} \bullet \mu_{2}\right)_{\ell} \leq 2^{\operatorname{dim} V-\rho} \operatorname{deg}\left(\mu_{1} \bullet \mu_{2}\right) .
\end{aligned}
$$

In view of the proof we have equality in (1.4) if $\rho \geq 0$ and in addition only the term with $\ell=\operatorname{dim} V$ occurs.

Example 4.3 Let $\mu_{1}$ and $\mu_{2}$ be the same $k$-plane $V$ in $\mathbb{P}^{n}$. Then $V \bullet V=V$, see [5, Section 1]. Thus only the term corresponding to $\ell=\operatorname{dim} V=k$ occurs in (4.5). If in addition $\rho \geq 0$, i.e., $2 k \geq n$, then each term in the expansion of $(1+\omega)^{\ell-\rho}$ gives a contribution and therefore, since $\ell=\operatorname{dim} V$,

$$
\operatorname{deg}(V \diamond V)=2^{\operatorname{dim} V-\rho} \operatorname{deg}(V \bullet V)
$$

so that the estimate (1.4) is sharp.

\section{The $\bullet L$-product on a projective manifold $Y$}

We shall now see that if $Y$ is projective and $L \rightarrow Y$ is a very ample line bundle, then there is an associated product $\bullet_{L}$ with the desired local multiplicities and a Bézout inequality for effective generalized cycle classes.

By definition 'very ample' means that there is an embedding

$$
i: Y \rightarrow \mathbb{P}^{M}
$$

such that $L=i^{*} \mathcal{O}_{\mathbb{P}^{M}}(1)$. For $\mu_{1}, \mu_{2} \in \mathcal{B}(Y)$, we define $\mu_{1} \bullet_{L} \mu_{2}$ as the unique element in $\mathcal{B}(Y)$ such that

$$
i_{*}\left(\mu_{1} \bullet L \mu_{2}\right)=i_{*} \mu_{1} \bullet i_{*} \mu_{2},
$$

where the right hand side is the $\bullet$-product in $\mathbb{P}^{M}$. We shall see that $\bullet_{L}$ only depends on $L$ and not on the embedding $i$.

Assume that $\mathbb{P}^{M}=\left\{\left[z_{0}, \ldots, z_{m}\right] ; z \in \mathbb{C}^{M+1}\right\}$. Since then $z_{0}, \ldots, z_{M}$ are global sections of $\mathcal{O}(1) \rightarrow \mathbb{P}^{M}$ it follows that $s_{k}:=i^{*} z_{k}, k=0, \ldots, M$, are in $H^{0}(Y, L)$. Moreover, $i$ is given by

$$
x \mapsto\left[s_{0}(x), \ldots, s_{M}(x)\right]
$$


Conversely, if we have $s_{0}, \ldots, s_{M}$ in $H^{0}(Y, L)$ such that (5.3) defines an embedding, then $i^{*} \mathcal{O}(1)=L$. In fact, since $g_{\ell k}=z_{\ell} / z_{k}$ are transition functions for $\mathcal{O}(1), s_{\ell} / s_{k}=i^{*} g_{\ell k}$ are transition functions for $i^{*} \mathcal{O}(1)$. Let

$$
N=\operatorname{dim} H^{0}(Y, L)-1 .
$$

Given an embedding (5.3) let us select a maximal linearly independent subset $s_{0}, \ldots, s_{N^{\prime}}$ of the $s_{k}$. Notice that then $N^{\prime} \leq N$. Let $i^{\prime}: Y \rightarrow \mathbb{P}^{N^{\prime}}$ be the embedding defined by these sections. Then, there is a linear subspace $\iota: V \rightarrow \mathbb{P}^{M}$ such that $i=\iota \circ i^{\prime}$. In view of [5, Proposition 6.7], $i$ and $i^{\prime}$ give rise to the same product on $Y$.

Thus we can assume that our embedding (5.1) is defined by (5.3), where $s_{0}, \ldots, s_{M}$ is a linearly independent set in $H^{0}(Y, L)$. In view of [5, Example 6.4] the product $\bullet_{L}$ only depends on the subspace of $H^{0}(Y, L)$ spanned by the given sections.

Proposition 5.1 Assume that we have the embedding (5.1) and let

$$
\hat{\rho}=\operatorname{dim} \mu_{1}+\operatorname{dim} \mu_{2}-M .
$$

Assume that $\mu_{1}, \mu_{2} \in \mathcal{B}(Y)$ have pure dimensions and let $d=\operatorname{dim} \mu_{1}+\operatorname{dim} \mu_{2}$ and $V=\left|\mu_{1}\right| \cap\left|\mu_{2}\right|$. Then

$$
\mu_{1} \diamond_{Y} \mu_{2}=\sum_{\ell=\max (0, \hat{\rho})}^{\operatorname{dim} V}\left(1+\omega_{L}\right)^{\ell-d-1} \wedge c(T Y) \wedge\left(\mu_{1} \bullet L \mu_{2}\right)_{\ell}
$$

and

$$
\mu_{1} \bullet L \mu_{2}=\sum_{k=0}^{\operatorname{dim} V}\left(1-\omega_{L}\right)^{k-d-1}\left(c(T Y)^{-1} \wedge \mu_{1} \diamond_{Y} \mu_{2}\right)_{k} .
$$

Since the right-hand side of (5.6) only depends on $L$, this holds for $\mu_{1} \bullet_{L} \mu_{2}$ as well.

Proof By Proposition 3.4 and (4.5) we have

$$
i_{*}\left(i^{*} c\left(N_{i(Y)} \mathbb{P}^{M}\right) \mu_{1} \diamond_{Y} \mu_{2}\right)=\sum_{\ell=\max (0, \hat{\rho})}^{\operatorname{dim} V}(1+\omega)^{\ell-\hat{\rho}}\left(i_{*} \mu_{1} \bullet i_{*} \mu_{2}\right)_{\ell} .
$$

Notice that since

$$
N_{i(Y)} \mathbb{P}^{M}=T \mathbb{P}^{M} / T(i(Y)),
$$

and $i^{*} \mathcal{O}(1)=L$, so that $i^{*} \omega=\omega_{L}$, we have that

$$
i^{*} c\left(N_{i(Y)} \mathbb{P}^{M}\right)=i^{*}\left(c\left(T \mathbb{P}^{M}\right) c\left(T(i(Y))^{-1}\right)=\left(1+\omega_{L}\right)^{M+1} c(T Y)^{-1}\right.
$$

on $Y$. Thus

$$
\left(1+\omega_{L}\right)^{M+1} c(T Y)^{-1} \wedge \mu_{1} \diamond_{Y} \mu_{2}=\sum_{\ell=\max (0, \hat{\rho})}^{\operatorname{dim} V}\left(1+\omega_{L}\right)^{\ell-\hat{\rho}}\left(\mu_{1} \bullet{ }_{L} \mu_{2}\right)_{\ell}
$$

which is the same as (5.5). Now (5.6) follows by Lemma 4.2.

Notice that there may occur negative powers of $1-\omega_{L}$ and $1+\omega_{L}$ in the sums (5.5) and (5.6).

Recall that if $\mu \in \mathcal{B}_{k}(Y)$, then, cf. (1.6),

$$
\operatorname{deg}_{L} \mu=\int_{Y} \mu \wedge \omega_{L}^{k}
$$


Proof of Theorem 1.2 Parts (i) and (ii) follow from Theorem 1.1 and (5.6).

Part (iii) follows from the corresponding statement for $\bullet=\bullet \bullet_{\mathbb{P}} M$ and (2.4). Alternatively, it follows from (5.6) and Theorem 1.1(ii).

Part (iv) follows from the analogous statement for $\bullet$ on $\mathbb{P}^{M}$. In fact, first notice that $\mu$ is effective if and only if $i_{*} \mu$ is. Then observe that if $\mu$ has pure dimension $k$, then

$$
\operatorname{deg}_{L} \mu=\int_{Y} \mu \wedge \omega_{L}^{k}=\int_{\mathbb{P}^{M}} i_{*} \mu \wedge \omega^{k}=\operatorname{deg}_{\mathbb{P}^{M}} i_{*} \mu .
$$

If $\mu_{1}$ and $\mu_{2}$ are effective, then $i_{*}\left(\mu_{1} \bullet L \mu_{2}\right)=i_{*} \mu_{1} \bullet i_{*} \mu_{2}$ is effective, see [5, Theorem 1.1], and hence $\mu_{1} \bullet L \mu_{2}$ is. From [5, Theorem 1.1] we thus have that

$\operatorname{deg}_{L}\left(\mu_{1} \bullet L \mu_{2}\right)=\operatorname{deg}_{\mathbb{P}^{M}}\left(i_{*} \mu_{1} \bullet i_{*} \mu_{2}\right) \leq \operatorname{deg}_{\mathbb{P}^{M}} i_{*} \mu_{1} \cdot \operatorname{deg}_{\mathbb{P}^{M}} i_{*} \mu_{2}=\operatorname{deg}_{L} \mu_{1} \cdot \operatorname{deg}_{L} \mu_{2}$

with equality if $\hat{\rho} \geq 0$.

Let us now consider (v). If $\mu_{1}$ and $\mu_{2}$ are cycles that intersect properly on $Y$, then by Theorem 1.1,

$$
\mu_{1} \diamond_{Y} \mu_{2}=\left(\mu_{1} \diamond_{Y} \mu_{2}\right)_{\rho}=\mu_{1} \cdot Y \mu_{2}
$$

where $\rho=\operatorname{dim} V=\operatorname{dim} \mu_{1}+\operatorname{dim} \mu_{2}-n$. From (5.6) we have

$$
\mu_{1} \bullet L \mu_{2}=\sum_{k=0}^{\rho}\left(1-\omega_{L}\right)^{k-d-1}\left(c(T Y)^{-1} \mu_{1} \diamond_{Y} \mu_{2}\right)_{k} .
$$

Now $k=\rho$ together with the term 1 from $\left(1-\omega_{L}\right)^{k-d-1}$ gives us $\mu_{1} \cdot Y \mu_{2}$, cf. (5.7). All other terms from $\left(1-\omega_{L}\right)^{k-d-1}$, or for $k<\rho$, will give contributions of strictly lower dimension, and they have vanishing multiplicities, see Sect. 2.3.

We have the following consequence of the proof.

Proposition 5.2 Let $M+1$ be the minimal dimension of a subspace $W$ of $H^{0}(Y, L)$ such that (5.3) is an embedding if $s_{0}, \ldots, s_{M}$ is a basis for $W$. If $\mu_{1}, \mu_{2} \in \mathcal{B}(Y)$ have pure dimensions and $\hat{\rho}=\operatorname{dim} \mu_{1}+\operatorname{dim} \mu_{2}-M \geq 0$, then

$$
\operatorname{deg}_{L}\left(\mu_{1} \bullet L \mu_{2}\right)=\operatorname{deg}_{L} \mu_{1} \cdot \operatorname{deg} \mu_{L} \mu_{2}
$$

\section{Some further properties}

In this section we still assume that $Y$ is a projective manifold. Assume that $\mu_{0}, \mu_{1} \in \mathcal{B}(Y)$ and that $\gamma$ is a smooth (closed) form in an open subset $U \subset Y$. We say that $\mu_{1}=\gamma \wedge \mu_{0}$ in $U$ if there are generalized cycles $\mu_{0}^{\prime}$ and $\mu_{1}^{\prime}$ representing $\mu_{0}$ and $\mu_{1}$, respectively, such that $\mu_{1}^{\prime}=\gamma \wedge \mu_{0}^{\prime}$ in $U$. We have the following version of Proposition 8.4 in [5].

Proposition 6.1 Assume that $\mu_{0}, \mu_{1}, \mu_{2} \in \mathcal{B}(Y), \gamma$ is smooth in the open set $U \subset Y$, and $\mu_{1}=\gamma \wedge \mu_{0}$ in $U$. Then

$$
\mu_{1} \diamond_{Y} \mu_{2}=\gamma \wedge\left(\mu_{0} \diamond_{Y} \mu_{2}\right)
$$

in $U$. If $L \rightarrow Y$ is a very ample line bundle, then

$$
\mu_{1} \bullet L \mu_{2}=\gamma \wedge\left(\mu_{0} \bullet L \mu_{2}\right)
$$

in $U$. 
Proof Fix suitable representatives $\mu_{0}^{\prime}, \mu_{1}^{\prime}, \mu_{2}^{\prime}$ in $\mathcal{G} \mathcal{Z}(Y)$ and a section $\eta$ that defines the diagonal $\Delta$ in $Y \times Y$. Moreover, let $\hat{c}\left(N_{\Delta}(Y \times Y)\right)$ be a fixed representative of the Chern class $c\left(N_{\Delta}(Y \times Y)\right)$. As usual, let $j: Y \rightarrow Y \times Y$ be the natural parametrization of $\Delta$. Then $j_{*}\left(\mu_{1} \diamond_{Y} \mu_{2}\right)$ is represented, cf. Sect. 2.6, by the generalized cycle

$$
j_{*}\left(\mu_{1}^{\prime} \diamond_{Y} \mu_{2}^{\prime}\right):=\hat{c}\left(N_{\Delta}(Y \times Y)\right) \wedge M^{\eta} \wedge\left(\mu_{1}^{\prime} \times \mu_{2}^{\prime}\right) .
$$

By assumption $\mu_{1}^{\prime}=\gamma \wedge \mu_{0}^{\prime}$ in $U$. Thus $\mu_{1}^{\prime} \times \mu_{2}^{\prime}=(\gamma \times 1) \wedge\left(\mu_{0}^{\prime} \times \mu_{2}^{\prime}\right)$ in $U \times U$. In view of [5, Example 2.7] therefore

$$
M^{\eta} \wedge\left(\mu_{1}^{\prime} \times \mu_{2}^{\prime}\right)=(\gamma \times 1) \wedge M^{\eta} \wedge\left(\mu_{0}^{\prime} \times \mu_{2}^{\prime}\right)
$$

in $U \times U$. By (6.3) and (6.4),

$$
\begin{aligned}
& j_{*}\left(\gamma \wedge\left(\mu_{0}^{\prime} \diamond_{Y} \mu_{1}^{\prime}\right)\right)=j_{*}\left(j^{*}(\gamma \times 1) \wedge\left(\mu_{0}^{\prime} \diamond_{Y} \mu_{1}^{\prime}\right)\right) \\
& \quad=(\gamma \times 1) \wedge j_{*}\left(\mu_{0}^{\prime} \diamond_{Y} \mu_{1}^{\prime}\right)=(\gamma \times 1) \wedge \hat{c}\left(N_{\Delta}(Y \times Y)\right) \wedge M^{\eta} \wedge\left(\mu_{0}^{\prime} \times \mu_{2}^{\prime}\right) \\
& \quad=\hat{c}\left(N_{\Delta}(Y \times Y)\right) \wedge M^{\eta} \wedge\left(\mu_{1}^{\prime} \times \mu_{2}^{\prime}\right)=j_{*}\left(\mu_{1}^{\prime} \diamond_{Y} \mu_{2}^{\prime}\right)
\end{aligned}
$$

in $U \times U$. Now (6.1) follows.

Assume that $\gamma$ has pure degree $v$. Then by (6.1),

$$
\left(c(T Y)^{-1} \mu_{1} \diamond_{Y} \mu_{2}\right)_{k}=\left(\gamma \wedge c(T Y)^{-1} \mu_{0} \diamond_{Y} \mu_{2}\right)_{k}=\gamma \wedge\left(c(T Y)^{-1} \mu_{0} \diamond_{Y} \mu_{2}\right)_{k+\nu} .
$$

Let $d$ be as in Proposition 5.1 and let $\tilde{d}=\operatorname{dim} \mu_{0}+\operatorname{dim} \mu_{2}$; then $d=\tilde{d}-v$. By (5.6),

$$
\begin{aligned}
\mu_{1} \bullet_{L} \mu_{2} & =\sum_{k \geq 0}\left(1-\omega_{L}\right)^{k-d-1}\left(c(T Y)^{-1} \mu_{1} \diamond_{Y} \mu_{2}\right)_{k} \\
& =\gamma \wedge \sum_{k \geq 0}\left(1-\omega_{L}\right)^{k+v-\tilde{d}-1}\left(c(T Y)^{-1} \mu_{0} \diamond_{Y} \mu_{2}\right)_{k+v} \\
& =\gamma \wedge \sum_{r \geq v}\left(1-\omega_{L}\right)^{r-\tilde{d}-1}\left(c(T Y)^{-1} \mu_{0} \diamond_{Y} \mu_{2}\right)_{r} \\
& =\gamma \wedge \sum_{r \geq 0}\left(1-\omega_{L}\right)^{r-\tilde{d}-1}\left(c(T Y)^{-1} \mu_{0} \diamond_{Y} \mu_{2}\right)_{r}=\gamma \wedge\left(\mu_{0} \bullet_{L} \mu_{2}\right),
\end{aligned}
$$

since the terms with $r<v$ in the last sum vanish when multiplied by $\gamma$.

We have the following version of Proposition 8.3 in [5].

Proposition 6.2 If $\mu \in \mathcal{B}(Y)$, then

$$
\mu \diamond_{Y} \mathbf{1}_{Y}=\mu
$$

If $a$ is a point in $Y$, then

$$
\mu \diamond_{Y}\{a\}=\operatorname{mult}_{a} \mu \cdot[a]
$$

and

$$
\mu \bullet_{L}\{a\}=\operatorname{mult}_{a} \mu \cdot[a] .
$$

Proof We can assume that $\mu=\tau_{*} \alpha$, where $\tau: W \rightarrow Y$ is proper and $\alpha$ is a product of components of first Chern forms. If $T=\tau \times 1: W \times Y \rightarrow Y \times Y$, then $T^{*} \mathcal{J}_{\Delta}$ is a regular embedding in $W \times Y$ since this sheaf defines the graph $G$ of $\tau$ in $W \times Y$. Notice that since $\left.N_{G}(W \times Y) \simeq T\right|_{G} ^{*} N_{\Delta}(Y \times Y)$,

$$
c\left(N_{G}(W \times Y)\right)=\left.T\right|_{G} ^{*} c\left(N_{\Delta}(Y \times Y)\right) .
$$


Moreover, by (2.6) we have

$$
S\left(\mathcal{J}_{\Delta}, \mu \otimes 1\right)=T_{*} S\left(T^{*} \mathcal{J}_{\Delta}, \alpha \otimes 1\right),
$$

and by [4, Proposition 1.4],

$$
c\left(N_{G}(W \times Y)\right) \wedge S\left(T^{*} \mathcal{J}_{\Delta}, 1_{W \times Y}\right)=[G] .
$$

By [4, Proposition 5.6] we have that $S\left(T^{*} \mathcal{J}_{\Delta}, \alpha \otimes 1\right)=(\alpha \otimes 1) \wedge S\left(T^{*} \mathcal{J}_{\Delta}, 1_{W \times Y}\right)$. Together with (6.8), (6.9), and (6.10) we have

$$
\begin{aligned}
& j_{*}\left(\mu \diamond_{Y} \mathbf{1}_{Y}\right)=c\left(N_{\Delta}(Y \times Y)\right) \wedge S\left(\mathcal{J}_{\Delta}, \mu \otimes 1\right)=T_{*}\left(c\left(N_{G}(W \times Y)\right) \wedge S\left(T^{*} \mathcal{J}_{\Delta}, \alpha \otimes 1\right)\right) \\
& =T_{*}\left((\alpha \otimes 1) \wedge c\left(N_{G}(W \times Y)\right) \wedge S\left(T^{*} \mathcal{J}_{\Delta}, 1_{W \times Y}\right)\right)=T_{*}((\alpha \otimes 1) \wedge[G]) .
\end{aligned}
$$

Let $\tilde{\tau}: W \rightarrow W \times Y, \tilde{\tau}(w)=(w, \tau(w))$, be the graph embedding. Then $(\alpha \otimes 1) \wedge[G]=\tilde{\tau}_{*} \alpha$ and $T \circ \tilde{\tau}=j \circ \tau$. Hence

$$
j_{*}\left(\mu \diamond_{Y} \mathbf{1}_{Y}\right)=T_{*}((\alpha \otimes 1) \wedge[G])=T_{*} \tilde{\tau}_{*} \alpha=j_{*} \tau_{*} \alpha=j_{*} \mu,
$$

which means that (6.5) holds.

The last two equalities can be verified in several ways. Notice that we can choose a neighborhood $U \subset Y$ of $a$ and coordinates $(x, y)$ in $U \times U$. Then $S\left(\mathcal{J}_{\Delta}, \mu \times\{a\}\right)$, restricted to $U \times U$, is represented by

$$
M^{x-y} \wedge(\mu \otimes[a])=\left(M^{x-a} \wedge \mu\right) \otimes[a]=\operatorname{mult}_{a} \mu \cdot[a] \otimes[a]=\operatorname{mult}_{a} \mu \cdot j_{*}[a],
$$

where the second equality follows from, e.g., [5, Equation (4.5)]. Hence, $S\left(\mathcal{J}_{\Delta}, \mu \times\{a\}\right)=$ $S_{0}\left(\mathcal{J}_{\Delta}, \mu \times\{a\}\right)$ and so

$$
j_{*}\left(\mu \diamond_{Y}\{a\}\right)=c\left(N_{\Delta}(U \times U) \wedge S\left(\mathcal{J}_{\Delta}, \mu \times\{a\}\right)=S_{0}\left(\mathcal{J}_{\Delta}, \mu \times\{a\}\right) .\right.
$$

Now (6.6) follows from (6.11). Since $\mu \diamond_{Y}\{a\}$ has dimension 0 it follows from (5.6) that $\mu \bullet L\{a\}=\mu \diamond_{Y}\{a\}$ and thus (6.7) follows.

Alternatively (6.7) follows from the definition (5.2) and the analogous statement for $\bullet=$ $\bullet_{\mathbb{P}} M$. Then (6.6) follows from (5.6) as above.

From (6.5) and (5.6) it follows that if $\mu$ has pure dimension, then

$$
\mu \bullet_{L} \mathbf{1}_{Y}=\sum_{k=0}^{\operatorname{dim} \mu}\left(1-\omega_{L}\right)^{k-n-\operatorname{dim} \mu-1}\left(c(T Y)^{-1} \wedge \mu\right)_{k},
$$

since $\operatorname{dim} V=\operatorname{dim} \mu$.

Let us now mention a possible way to express our products as limits of smooth forms times $\mu_{1} \times \mu_{2}$. It follows from [4, Proposition 5.7] that the representatives $M_{k}^{\sigma} \wedge \hat{\mu}$ of $S_{k}(\mathcal{J}, \mu)$, cf. (2.5), can be computed by the formula

$$
M_{k}^{\sigma} \wedge \hat{\mu}=\lim _{\epsilon \rightarrow 0} \frac{\epsilon\left(d d^{c}|\sigma|^{2}\right)^{k}}{\left(|\sigma|^{2}+\epsilon\right)^{k+1}} \wedge \hat{\mu}, \quad k=0,1, \ldots
$$

In particular we have

Proposition 6.3 If $\mu_{1}, \mu_{2} \in \mathcal{B}(Y)$ are represented by $\hat{\mu}_{1}, \hat{\mu}_{2}$, and $\eta$ is a section of a Hermitian bundle $E \rightarrow Y \times Y$ that defines $\mathcal{J}_{\Delta}$, then $j_{*}\left(\mu_{1} \diamond_{Y} \mu_{2}\right)$ is represented by the limits

$$
c\left(N_{\Delta}(Y \times Y)\right) \wedge \sum_{k=0}^{\operatorname{dim} V} \lim _{\epsilon \rightarrow 0} \frac{\epsilon\left(d d^{c}|\eta|^{2}\right)^{k}}{\left(|\eta|^{2}+\epsilon\right)^{k+1}} \wedge\left(\hat{\mu}_{1} \times \hat{\mu}_{2}\right) .
$$


One gets a formula for $\mu_{1} \diamond_{Y} \mu_{2}$ by taking $\pi_{*}$ of (6.14), where $\pi: Y \times Y \rightarrow Y$ is the projection onto the first (or the second) factor, since $\pi \circ j=I d_{Y}$. One can get similar formulas for $\mu_{1} \bullet_{L} \mu_{2}$ by combining (6.14) and (5.6).

\section{Examples}

We first recall the so-called Segre embedding

$$
i: \mathbb{P}_{x}^{m} \times \mathbb{P}_{y}^{n} \rightarrow \mathbb{P}^{(m+1)(n+1)-1} .
$$

Let $Y=\mathbb{P}_{x}^{m} \times \mathbb{P}_{y}^{n}$ and consider the line bundle $L=\mathcal{O}(1)_{\mathbb{P}_{x}^{m}} \otimes \mathcal{O}(1)_{\mathbb{P}_{y}^{n}}$. The set of sections $\left\{x_{j} y_{k} ; 0 \leq j \leq m, 0 \leq k \leq n\right\}$ is a basis for $H^{0}(Y, L)$ and $i$ is the associated embedding, cf. Sect. 5 ,

$$
([x],[y]) \mapsto\left[x_{0} y_{0}: \ldots: x_{0} y_{n}: \ldots: x_{m} y_{0}: \ldots: x_{m} y_{n}\right]
$$

Notice that

$$
i^{*} \omega=\omega_{L}=\omega_{x}+\omega_{y},
$$

where $\omega, \omega_{x}$ and $\omega_{y}$ are (representatives of) $c_{1}\left(\mathcal{O}(1)_{\mathbb{P}^{(m+1)(n+1)-1}}\right), c_{1}\left(\mathcal{O}(1)_{\mathbb{P}_{x}^{m}}\right)$, and $c_{1}\left(\mathcal{O}(1)_{\mathbb{P}_{y}^{n}}\right)$, respectively.

We now consider the self-intersection of an exceptional divisor.

Example 7.1 Consider the blowup $Y=B l_{p} \mathbb{P}^{2}$ of $\mathbb{P}^{2}$ at the point $p=[1: 0: 0]$, and let both $\mu_{1}$ and $\mu_{2}$ be the exceptional divisor $E$. We have the embedding

$$
j: Y \rightarrow Y^{\prime}:=\mathbb{P}_{x}^{2} \times \mathbb{P}_{y}^{1}
$$

so that $j(Y)=\{f=0\}$, where $f$ is the section $f=x_{1} y_{1}-x_{2} y_{0}$ of $\mathcal{O}(1)_{\mathbb{P}_{x}^{2}} \otimes \mathcal{O}(1)_{\mathbb{P}_{y}^{1}}$. Let $L \rightarrow Y$ be the pullback to $Y$ of this line bundle, which we for simplicity denote in the same way so that

$$
\omega_{L}=\omega_{x}+\omega_{y} .
$$

We now compose with the Segre embedding

$\sigma: \mathbb{P}_{x}^{2} \times \mathbb{P}_{y}^{1} \rightarrow \mathbb{P}_{z}^{5}, \quad\left(\left[x_{0}: x_{1}: x_{2}\right],\left[y_{0}: y_{1}\right]\right) \mapsto\left[x_{0} y_{0}: x_{0} y_{1}: x_{1} y_{0}: x_{1} y_{1}: x_{2} y_{0}: x_{2} y_{1}\right]$ and get the embedding $i=\sigma \circ j: Y \rightarrow \mathbb{P}^{5}$. We claim that

$$
E \bullet{ }_{L} E=E
$$

and

$$
E \diamond_{Y} E=E-\omega_{L} \wedge E .
$$

In fact, the image of $E$ in $\mathbb{P}^{2} \times \mathbb{P}^{1}$ is $\{[1: 0: 0]\} \times \mathbb{P}_{y}^{1}$ so the image in $\mathbb{P}^{5}$ is the line $\left\{\left[y_{0}: y_{1}: 0: 0: 0: 0\right]\right\}$. Therefore $i_{*} E \bullet i_{*} E=i_{*} E$, see the remark after [5, Theorem 1.1], and thus (7.2) holds. Next we compute $E \diamond_{Y} E$. In view of (7.2) only the term with $\ell=1$ occurs in (5.5) and since $\ell-d-1=1-2-1=-2$ we have

$$
E \diamond_{Y} E=\left(1+\omega_{L}\right)^{-2} \wedge c(T Y) \wedge E .
$$

Now, cf. [4, Eq (7.5)], $c\left(N_{Y} Y^{\prime}\right)=c(L)=1+\omega_{x}+\omega_{y}$ and thus

$$
c(T Y)=c\left(T Y^{\prime}\right) / c\left(N_{Y} Y^{\prime}\right)=\left(1+\omega_{x}\right)^{3}\left(1+\omega_{y}\right)^{2} /\left(1+\omega_{x}+\omega_{y}\right) .
$$


Since $\omega_{x}=0$ on $E$ we have, cf. (7.1),

$$
E \diamond_{Y} E=\frac{1}{1+\omega_{y}} \wedge E=\left(1-\omega_{y}\right) \wedge E=\left(1-\omega_{L}\right) \wedge E .
$$

Let us next look at an example where $Y$ is embedded into $\mathbb{P}^{M}$ for a minimal $M$, and where the terms $\cdots$ of lower dimension in (1.9) do not vanish.

Example 7.2 Let $Y=\mathbb{P}_{x}^{1} \times \mathbb{P}_{y}^{1}$ and let

$$
i: Y \rightarrow \mathbb{P}^{3}, \quad\left(\left[x_{0}: x_{1}\right],\left[y_{0}: y_{1}\right]\right) \mapsto\left[x_{0} y_{0}: x_{0} y_{1}: x_{1} y_{0}: x_{1} y_{1}\right],
$$

be the Segre embedding. Note that $Y \cdot_{Y} Y=Y$ since it is a proper intersection. It follows from Theorem 1.1 that

$$
Y \diamond_{Y} Y=Y .
$$

We want to compute $Y \bullet_{L} Y$. Since $\omega_{L}=i^{*} \omega=\omega_{x}+\omega_{y}$, cf. (7.1), it follows that

$$
\omega_{L}^{2}=\left(\omega_{x}+\omega_{y}\right)^{2}=2 \omega_{x} \wedge \omega_{y}
$$

and thus

$$
\operatorname{deg}_{L} Y=\int_{Y} \omega_{L}^{2}=\int_{\mathbb{P}_{x}^{1} \times \mathbb{P}_{y}^{1}} 2 \omega_{x} \wedge \omega_{y}=2 .
$$

Since $\hat{\rho}=\operatorname{dim} Y+\operatorname{dim} Y-3=1 \geq 0$, the Bézout formula

$$
\operatorname{deg}_{L}\left(Y \bullet_{L} Y\right)=\operatorname{deg}_{L} Y \cdot \operatorname{deg}_{L} Y
$$

holds, cf. Proposition 5.2. Thus $Y \bullet_{L} Y$ must have degree $2 \cdot 2=4$. On the other hand, $\cdots$ in (1.9) can only contain a term $\mu$ of dimension $\hat{\rho}=1$, since all components of $i_{*} Y \bullet i_{*} Y$ must have dimension at least the expected dimension $\hat{\rho}$, cf. (1.5) and Sect. 4. Thus $\operatorname{deg}_{L} \mu=2$. For symmetry reasons it is natural to guess that

$$
Y \bullet{ }_{L} Y=Y+\omega_{L} \wedge Y .
$$

Let us check (7.5) by means of (5.5) in Proposition 5.1 and (7.4).

Notice that $d=\operatorname{dim} Y+\operatorname{dim} Y=4, \hat{\rho}=d-3=1$, and $V=Y$ so that $\operatorname{dim} V=2$. Moreover,

$$
\begin{aligned}
c(T Y) & =c\left(T \mathbb{P}_{x}^{1}\right) \wedge c\left(T \mathbb{P}_{y}^{1}\right)=\left(1+\omega_{x}\right)^{2} \wedge\left(1+\omega_{y}\right)^{2}=\left(1+2 \omega_{x}\right) \wedge\left(1+2 \omega_{y}\right) \\
& =1+2\left(\omega_{x}+\omega_{y}\right)+4 \omega_{x} \wedge \omega_{y}=1+2 \omega_{L}+2 \omega_{L}^{2} .
\end{aligned}
$$

Assuming (7.5), the right hand side of (5.5) equals

$$
\begin{aligned}
& c(T Y) \wedge \sum_{\ell=1}^{2}\left(1+\omega_{L}\right)^{\ell-5} \wedge\left(Y+\omega_{L} \wedge Y\right)_{\ell}=c(T Y) \wedge\left(\frac{1}{\left(1+\omega_{L}\right)^{4}} \wedge \omega_{L} \wedge Y+\frac{1}{\left(1+\omega_{L}\right)^{3}} \wedge Y\right) \\
& =c(T Y) \wedge \frac{1}{\left(1+\omega_{L}\right)^{4}}\left(\omega_{L}+1+\omega_{L}\right) \wedge Y=\frac{1}{\left(1+\omega_{L}\right)^{4}}\left(1+2 \omega_{L}+2 \omega_{L}^{2}\right) \wedge\left(1+2 \omega_{L}\right) \wedge Y \\
& =\frac{1}{1+4 \omega_{L}+6 \omega_{L}^{2}}\left(1+4 \omega_{L}+6 \omega_{L}^{2}\right) \wedge Y=Y=Y \diamond_{Y} Y .
\end{aligned}
$$

Hence our guess is correct. Clearly one can just as well start with (7.4) and apply (5.6). By similar computations one then gets (7.5), as expected. 
The following example, which is an elaboration of [5, Example 8.10], shows that the product $\diamond_{Y}$ is not associative.

Example 7.3 Consider the hypersurface $Z=\left\{x_{2} x_{1}^{m}-x_{3}^{2} x_{0}^{m-1}=0\right\}$ and the hyperplanes $H_{2}=\left\{x_{2}=0\right\}$ and $H_{3}=\left\{x_{3}=0\right\}$ in $Y=\mathbb{P}^{3}$. (All products here are taken in $\mathbb{P}^{3}$; let $\diamond=\diamond_{\mathbb{P}^{3}}$ and $\cdot=\cdot_{\mathbb{P}^{3}}$.) Since $H_{2}$ and $Z$ intersect properly,

$$
H_{2} \diamond Z=H_{2} \bullet Z=H_{2} \cdot Z=2\left\{x_{2}=x_{3}=0\right\}+(m-1)\left\{x_{0}=x_{2}=0\right\}=: 2 A+(m-1) B .
$$

Moreover, since $\mathrm{H}_{3}$ and $B$ intersect properly,

$$
H_{3} \diamond B=H_{3} \cdot B=[b],
$$

where $b=[0,1,0,0]$. In [5, Example 8.10] is showed that $H_{3} \bullet A=A$. In view of (4.5) it follows that

$$
H_{3} \diamond A=(1+\omega) \wedge A .
$$

Indeed $\rho=\operatorname{dim} H_{3}+\operatorname{dim} A-n=2+1-3=0$ and $H_{3} \bullet A=A$ has pure dimension 1 . By (7.6)-(7.8) we conclude

$$
H_{3} \diamond\left(H_{2} \diamond Z\right)=2(1+\omega) \wedge A+(m-1)[b] .
$$

Next, since $H_{3}$ and $H_{2}$ intersect properly, it follows that $H_{3} \diamond H_{2}=H_{3} \cdot H_{2}=A$. In [5, Example 8.10] we showed that $A \bullet Z=A+m[a]$, where $a=[1: 0: 0: 0]$. As above, we compute $A \diamond Z$ by applying (4.5). To do this, note that $\rho=\operatorname{dim} A+\operatorname{dim} Z-n=1+2-3=0$ and $V=|A| \cap|Z|=|A|$, so that $\operatorname{dim} V=1$. Thus

$$
\left(H_{3} \diamond H_{2}\right) \diamond Z=\sum_{\ell=0}^{1}(1+\omega)^{\ell} \wedge(A \bullet Z)_{\ell}=m[a]+(1+\omega) \wedge A .
$$

Clearly the right hand sides in (7.9) and (7.10) are different in $\mathcal{B}\left(\mathbb{P}^{3}\right)$.

Acknowledgements We would like to thank Bo Berndtsson, Martin Raum and Jan Stevens for valuable discussions on questions in this paper. We would also like to thank the referee for important comments and suggestions.

Funding Open access funding provided by Chalmers University of Technology.

Open Access This article is licensed under a Creative Commons Attribution 4.0 International License, which permits use, sharing, adaptation, distribution and reproduction in any medium or format, as long as you give appropriate credit to the original author(s) and the source, provide a link to the Creative Commons licence, and indicate if changes were made. The images or other third party material in this article are included in the article's Creative Commons licence, unless indicated otherwise in a credit line to the material. If material is not included in the article's Creative Commons licence and your intended use is not permitted by statutory regulation or exceeds the permitted use, you will need to obtain permission directly from the copyright holder. To view a copy of this licence, visit http://creativecommons.org/licenses/by/4.0/.

\section{References}

1. Achilles, R., Manaresi, M.: Multiplicities of a bigraded ring and intersection theory. Math. Ann. 309(4), 573-591 (1997)

2. Achilles, R., Rams, S.: Intersection numbers, Segre numbers and generalized Samuel multiplicities. Arch. Math. (Basel) 77(5), 391-398 (2001)

3. Andersson, M., Samuelsson Kalm, H., Wulcan, E., Yger, A.: Segre numbers, a generalized King formula, and local intersections. J. Reine Angew. Math. 728, 105-136 (2017) 
4. Andersson, M., Eriksson, D., Samuelsson Kalm, H., Wulcan, E., Yger, A.: Global representation of Segre numbers by Monge-Ampère products. Math. Ann. 380, 349-391 (2021)

5. Andersson, M., Eriksson, D., Samuelsson Kalm, H., Wulcan, E., Yger, A.: Non-proper intersection products and generalized cycles. Eur. J. Math. https://doi.org/10.1007/s40879-021-00473-W

6. Flenner, H., O’Carroll, L., Vogel, W.: Joins and Intersections. Springer Monographs in Mathematics. Springer, Berlin (1999)

7. Fulton, W.: Intersection Theory, 2nd edn. Springer, Berlin (1998)

8. Gaffney, T., Gassler, R.: Segre numbers and hypersurface singularities. J. Algebraic Geom. 8(4), 695-736 (1999)

9. van Gastel, L.J.: Excess intersections and a correspondence principle. Invent. Math. 103(1), 197-222 (1991)

10. Samuelsson Kalm, H.: A pullback operation on a class of currents. arXiv:2004.08165

11. Stückrad, J., Vogel, W.: An algebraic approach to the intersection theory. In: The Curves Seminar at Queens, vol. II (Kingston, Ont., 1981/1982), Exp. No. A, 32. Queen's Papers in Pure and Applied Mathematics, vol. 61. Queen's University, Kingston (1982)

12. Tworzewski, P.: Intersection theory in complex analytic geometry. Ann. Pol. Math. 62(2), 177-191 (1995)

13. Vogel, W.: Lectures on Results on Bézout's Theorem, Notes by D. P. Patil. Tata Institute of Fundamental Research Lectures on Mathematics and Physics, vol. 74. Published for the Tata Institute of Fundamental Research, Bombay; by Springer, Berlin (1984)

14. Yger, A.: Flexibility of Analysis Serving Computational Polynomial Algebra or Arithmetics. Complex Anal. Oper. Theory 15, 61 (2021). https://doi.org/10.1007/s11785-021-01080-z

Publisher's Note Springer Nature remains neutral with regard to jurisdictional claims in published maps and institutional affiliations. 